\title{
Prognostic significance of TEM7 and nestin expression in women with advanced high grade serous ovarian cancer
}

\author{
Artur Czekierdowski ${ }^{1}$, Norbert Stachowicz ${ }^{2}$, Sylwia Czekierdowska ${ }^{1}$, Tomasz Łoziński ${ }^{3}$, \\ Grzegorz Gurynowicz ${ }^{4}$, Tomasz Kluz ${ }^{5}$ \\ ${ }^{1}$ Department of Gynecological Oncology and Gynecology, Medical University of Lublin, Poland \\ ${ }^{2}$ Chair and Department of Epidemiology and Clinical Research Methodology, Medical University of Lublin, Poland \\ ${ }^{3}$ Department of Obstetrics and Gynecology, Pro-Familia Hospital, Rzeszow, Poland \\ ${ }^{4}$ Division of Perinatology and Women's Diseases, Poznan University of Medical Sciences, Poznan, Poland \\ ${ }^{5}$ Department of Obstetrics and Gynecology, Fryderyk Chopin University Hospital No 1, Faculty of Medicine, \\ Rzeszow University, Poland
}

\begin{abstract}
Objectives: Tumor endothelial marker 7 (TEM7) and nestin have been proposed to be new candidates for neoangiogenesis assessment. Nestin is also cancer stem cells marker in various malignant tumors. AIMS. To investigate the expression of TEM7, nestin and nestin-related microvessel density (MVD) in high-grade serous ovarian cancer samples and to study their correlation with overall survival (OS) and disease-free survival (DFS) times.

Material and methods: Tumor samples obtained from 70 women with FIGO IIIc/IV ovarian serous cancer were studied with immunohistochemistry.

Results: Patients median age was 54 yrs (range: $29-72$ years), $86 \%$ died of the disease with median OS $=28.5$ months and median DFS $=10$ months (3 years DFS $=19 \% ; 5$ years. DFS $=13.8 \%$ ). High nestin expression was found in 16 ( $23 \%$ ) patients with 3 years and 5 years OS of $14 \%$ and $0 \%$. In low-nestin expression group OS and DFS were $42 \%$ and $25 \%$, respectively. Median nestin-MVD (16, range:12-23) was not correlated with cancer cells nestin expression and with both DFS and OS. High TEM7 expression was found in 29 women (41\%) of whom 21 (72\%) died of the disease. A 5-year OS in these women was $27 \%$ as compared to $8 \%$ in low TEM7 expression group, but TEM7 presence had no association with nestin, nestin-MVD and both OS and DFS.
\end{abstract}

Conclusions: Nestin as a marker of cancer stem cells may assist in the prediction of OS and DFS in women with high grade serous ovarian cancer. Nestin may also be considered a novel therapeutic target for antiangiogenic agents.

Key words: High grade serous ovarian cancer, TEM-7, nestin, disease-free survival, overall survival

Ginekologia Polska 2018; 89, 3: 135-141

\section{INTRODUCTION}

Ovarian epithelial cancer has the highest mortality rate of all gynecological malignancies and the vast majority of the ovarian cancer deaths is related to advanced clinical stages of high-grade serous ovarian carcinoma (HGSOC) [1]. Most cases of HGSOC possess a relatively simple mutational profile with TP53 nearly always mutated and with other genes mutated at low frequency [2]. During last two decades only a small improvement in overall survival has been achieved. Since HGSOC is a molecularly and clinically heterogeneous disease, this perspective may not be valid any more in the era of personalized therapy, and given that high-grade serous cancer is extremely sensitive and other subtypes are relatively resistant to chemotherapy [3-5]. 
Targeting tumor angiogenesis has been validated as a therapeutic strategy in the treatment of different malignancies, including high grade and recurrent ovarian cancer [6, 7]. An expanded research dedicated to discover new agents capable of targeting the endothelial layer of malignant tumors microvessels has resulted in discovery of the TEM proteins family and nestin $[8,9]$

Nestin belongs to intermediate filament proteins group and since its discovery was believed to be associated solely with neural progenitor cells. Studies performed later revealed that this protein may also be involved in malignant tumors neoangiogenesis and more recently nestin has been also documented to be one of cancer stem cells markers [10]. Although nestin is expressed at the early stages of normal human development it was also found in several physiological conditions like e.g. in tissue repair processes through proliferating vascular endothelial cells or in various malignant tumors. Previous studies showed that nestin could be another specific indicator of ovarian cancer aggressive and clinically advanced forms [11,12]. Correlation between nestin expression in tumor tissue and the clinical course of the disease was reported for breast and ovarian cancers, as well as for gastrointestinal tumors, germ cell tumors, osteosarcomas and melanomas [10]. In some tumor types, increased nestin expression correlates with the tumor grade and indicates an immature and invasive phenotype of transformed cells [11, 12]. Interestingly, the use of nestin as a predictive marker of a poor response to conventional therapy and to novel therapeutic agents was reported for multiple myeloma [13].

A novel group of Tumor Endothelial Markers (TEMs) could be potentially used as new markers for immunohistochemical assessment of microvessel network formation in various cancers $[8,14]$. TEMs are proteins found in various malignant tumors tissue with much higher expression when compared to endothelial cells of microvessels found in healthy tissues. Important members of TEM family, and also the most promising candidates indicating cancer neoangiogenesis markers are: TEM1, TEM5, TEM7, and TEM8 $[14,15]$. Special attention has been given to TEM7, a transmembrane protein that can also be found as a bound form, as well as soluble and secreted forms $[14,16]$. Tumor endothelial marker 7 (TEM7) was initially identified as the most abundantly expressed gene in a group of cell surface proteins in the vascular endothelium of human tumors $[8,17,18]$. Overexpression of TEMs, and in particular TEM7 in ovarian cancer compared to benign masses and normal ovarian tissue suggests its possible role in tumor microvessel formation [14]. Because of these properties, TEMs appear to be perspective candidates for early detection, monitoring, and treatment of aggressive forms of EOC.
The aims of this study were to investigate the expression of TEM-7, nestin and nestin-related microvessel density (MVD) in high-grade serous ovarian cancer samples and to examine their correlation with overall survival (OS) and disease-free survival (DFS) times of affected women.

\section{MATERIAL AND METHODS \\ Patients and tumor samples}

Clinical and demographical data were collected from a group of 70 patients with FIGO stage IIIc/IV of high grade ovarian serous cancer. Samples of cancer tissues were acquired following surgery and prior to chemotherapy from the Department of Molecular Pathology of the Maria Sklodowska-Curie Memorial Cancer Center and Institute of Oncology, Warsaw, Poland. The material was selected out of 199 women with serous ovarian cancer who were treated in this hospital between 2000-2005. The clinical characteristics of these patients with p53 mutational status and outcome data following standardized chemotherapy regimens have been previously described [19]. All women in the present study met several inclusion criteria, among them an adequate staging procedure that included total hysterectomy with bilateral salpingo-oophorectomy and omentectomy, aspiration of ascites or multiple cytologic washings, and multiple peritoneal biopsies including the diaphragm. In all cases complete abdominal exploration followed by pelvic and paraaortic lymph node sampling or complete paraaortic lymphadenectomy was performed. In all women 3-years and 5 years OS and DSF rates were calculated and compared to their menopausal status, residual tumor size, chemotherapy sensitivity, the $\mathrm{p} 53$ protein mutational status and next with TEM7 and nestin expression as well as nestin microvascular density (MVD).

Imunohistochemical staining procedure was performed on paraffin-embedded tissues probe cut in $5 \mu \mathrm{m}$-thick sections and placed on silanized slides according to method described previously [12]. Tumor tissue slides were incubated with the primary anti-nestin antibody (Sigma-Aldrich) (1:150) or anti-TEM7 antibody (Sigma-Aldrich) (1:200) for $60 \mathrm{~min}$ at room temperature. The DAKO rabbit/mouse EnVision $\odot+$ HRP System were used for detection. Sections were counterstained with hematoxylin. To ensure tissue quality basic routine $\mathrm{H}+\mathrm{E}$ staining was performed for all specimens.

The average number of nestin+ microvessels within selected tumor areas was measured according to standard Weidner's MVD scoring method as previously described [12]. To identify the most vascularized areas of the tumors with the highest density of nestin + microvessels sections were examined at low magnifications ( $\times 40$ and $\times 100)$. Then, as a rule, 10 fields were examined, except in a few cases where less tumor tissue was available. The mean numbers 
of vessels were expressed as the average of all fields examined at high magnification $(\times 200)$; per high power field (nestin-MVD/HPF). Nestin and TEM7 expression in tumor cells was evaluated semi-quantitatively. Intensity of staining reaction and percentage of stain-positive cells were measured under $\times 200$ magnification. The intensity of staining was classified " 0 " for absent staining, "1" for weak, " 2 " for moderate, and " 3 " for intense reaction. The proportion of stain-positive cells was visually calculated and cell expression was stratified as follows: low; for $<20 \%$ positive cells (weak or absent staining reaction), medium $21-50 \%$ positive cells (weak or moderate staining reaction) and high > 51\% of positive cells (moderate or strong staining reaction).

\section{Statistical analysis}

Statistical analysis was performed with the use of Statistica v.10.0 software (Statsoft, Poland). Association between categorical variables was examined by Pearson's $X^{2}$ test or Fisher's exact tests, where appropriate. Mann-Whitney nonparametric test was used to compare categorical with continuous tumor variables when there were two categories, whereas Kruskal-Wallis nonparametric test was used instead, when there were more than two categories. Overall survival (OS) and disease-free survival (DFS) was estimated using the Kaplan-Meier method. We analyzed the 3-year and 5-year survival rates of patient groups with different TEM7 and nestin-related parameters. OS and DFS were calculated with the multivariate Cox' proportional hazards model. The criterion of statistical significance applied in all calculations was $p<0.05$. The study was approved by the Medical University of Lublin Bioethical Committee.

\section{RESULTS}

Selected characteristics of the studied ovarian cancer patient cohort are presented in Table 1. Patients median age was 54 years. (range: $29-72$ years), $86 \%$ died of the disease and with median overall survival time was 28.5 months and median DFS time was 10 months. As expected, the probability of survival was higher if the residual tumor size was smaller or complete cytoreduction was achieved. Chemotherapy sensitivity was another statistically significant and important predictive factor for OS and DSF. The results of survival analysis according to clinical futures are shown in Table 1. Figures 1 and 2 present the typical nestin staining pattern in ovarian cancer tissues. Nestin protein was predominantly localized in the cytoplasm of cancer cells and also in endothelial cells around small capillaries. Figures 3 and 4 show typical expression pattern of TEM7 in tumor cells. Table 2 presents OS and DSF rates compared to TEM/nestin expression and according to nestin microvessel density. There were statistically significant differences in OS and DSF rates between groups with low and high nestin expression in tumor cells ( $p=0.02$ and $p=0.01$, respectively). High nestin expression was found in 14 patients. In this group OS was $14 \%$ after 3 years and $0 \%$ after 5 years. Corresponding DSF rates in these

Table 1. Selected clinical features overall survival (OS) rates and disease free survival (DSF) in the studied group of women with HGSOC

\begin{tabular}{|c|c|c|c|c|c|c|c|}
\hline & $\begin{array}{l}\mathrm{N} \text { (no. of } \\
\text { patients) }\end{array}$ & $\begin{array}{c}\text { OS } \\
\text { 3-year }\end{array}$ & $\begin{array}{c}\text { OS } \\
\text { 5-year }\end{array}$ & p & $\begin{array}{c}\text { DSF } \\
\text { 3-year }\end{array}$ & $\begin{array}{c}\text { DSF } \\
\text { 5-year }\end{array}$ & $\mathbf{P}$ \\
\hline All group & 70 & $37 \%$ & $22 \%$ & & $18 \%$ & $15 \%$ & \\
\hline \multicolumn{8}{|l|}{ Residual tumor size } \\
\hline 0 & 11 & $54 \%$ & $27 \%$ & \multirow{5}{*}{$p=0.03$} & $46 \%$ & $35 \%$ & \multirow{5}{*}{$p=0.009$} \\
\hline$<0.5 \mathrm{~cm}$ & 5 & $46 \%$ & $22 \%$ & & $16 \%$ & $16 \%$ & \\
\hline $0.5-2 \mathrm{~cm}$ & 16 & $31 \%$ & $15 \%$ & & $14 \%$ & $14 \%$ & \\
\hline $2-5 \mathrm{~cm}$ & 4 & $50 \%$ & $25 \%$ & & $25 \%$ & $25 \%$ & \\
\hline $5 \mathrm{~cm}$ & 24 & $25 \%$ & $13 \%$ & & $6 \%$ & $6 \%$ & \\
\hline \multicolumn{8}{|l|}{ P53 mutation } \\
\hline No & 27 & $16 \%$ & $16 \%$ & \multirow{2}{*}{$p=0.12$} & $14 \%$ & $14 \%$ & \multirow{2}{*}{$p=0.51$} \\
\hline Yes & 43 & $49 \%$ & $26 \%$ & & $19 \%$ & $16 \%$ & \\
\hline \multicolumn{8}{|l|}{ Menopausal status } \\
\hline Before MNP & 27 & $50 \%$ & $25 \%$ & \multirow{2}{*}{$p=0.13$} & $27 \%$ & $22 \%$ & \multirow{2}{*}{$p=0.21$} \\
\hline After MNP & 43 & $29 \%$ & $20 \%$ & & $11 \%$ & $11 \%$ & \\
\hline \multicolumn{8}{|c|}{ Chemotherapy treatment sensitivity } \\
\hline Resistant & 34 & $21 \%$ & $8 \%$ & \multirow{3}{*}{$p=0.00001$} & $0 \%$ & $0 \%$ & \multirow{3}{*}{$p<0.0005$} \\
\hline Moderatly sensitive & 24 & $27 \%$ & $0 \%$ & & $5 \%$ & $5 \%$ & \\
\hline Highly sensitive & 12 & $91 \%$ & $83 \%$ & & $75 \%$ & $65 \%$ & \\
\hline
\end{tabular}




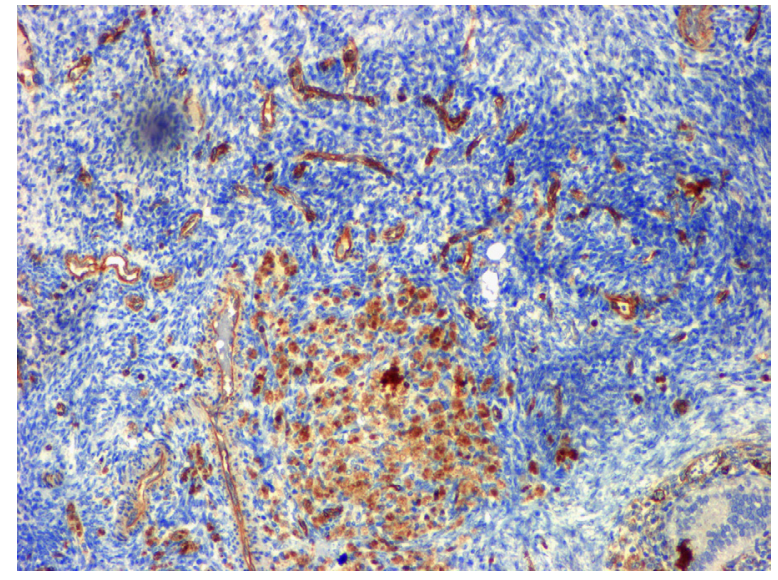

Figure 1. Nestin staining in ovarian cancer tissues. Nestin protein is predominantly localized in cytoplasm of endothelial cells around small capillaries (open arrows) and in tumor cells (full arrows). Magnification: x100

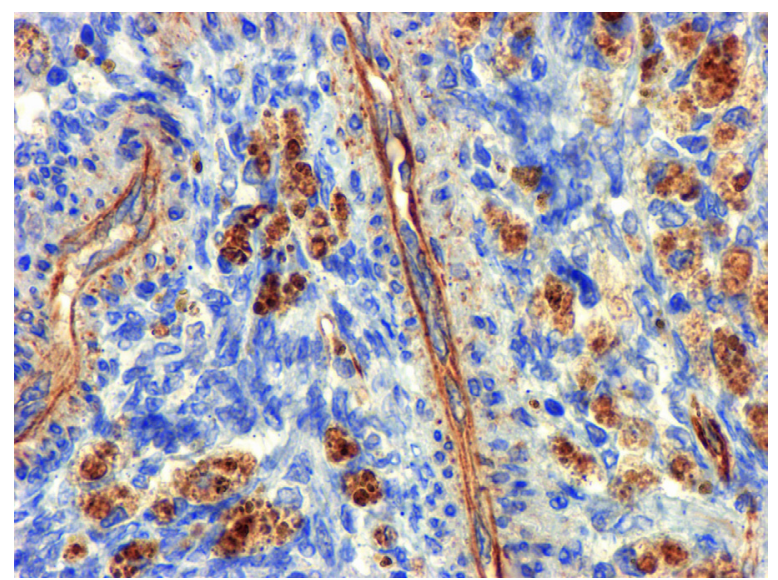

Figure 2. Selected area of nestin staining of ovarian cancer cells (full arrow) and microcapillaries (open arrow). Magnification: x400

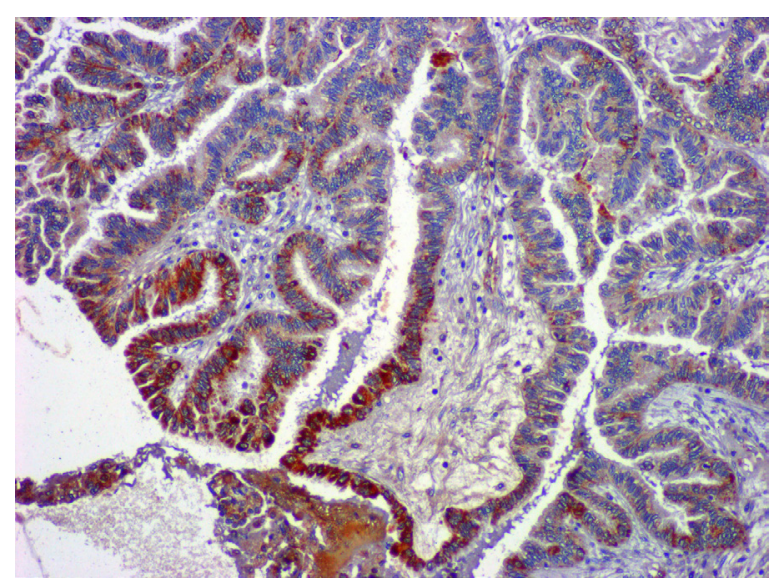

Figure 3. Expression of TEM-7 in tumor cells of High Grade Serous Ovarian Cancer. Magnification: x100

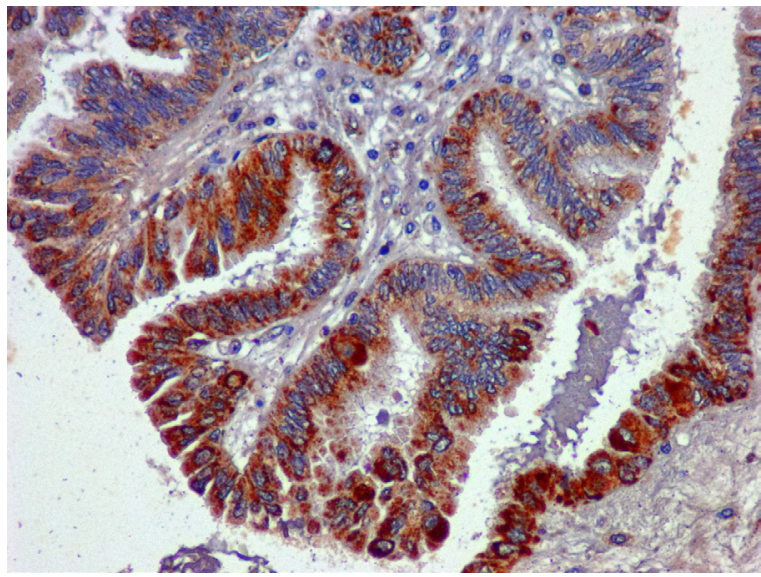

Figure 4. Selected area of Fig. 3 with TEM-7 expression in cancer cells of High Grade Serous Ovarian Cancer. Magnification: x200

women were $9 \%$ and $0 \%$ after 3 and 5 years, respectively. In the group with low or medium nestin expression OS rates were more than $40 \%$ after 3 years and more than $20 \%$ after 5 years (Tab. 2). Nestin-MVD (median 16, range: 12-23) did not correlate with nestin expression in cancer cells. OS and DSF rates were not significantly different when high and low nestin density in tumor microvessels was considered (Tab. 2). High TEM7 expression was found in tumor samples of 29 women (41\%) of whom 21 (72\%) died of the disease. In the group of women with high TEM7 tumor expression a 5 -year OS was $25 \%$ and it was not statistically different from the group of women with low TEM7 tumor expression in whom OS was $21 \%$ after 5 years. DSF rate in women with low TEM7 tumor expression was $8 \%$ and, although lower than in the high TEM7 expression group where DSF was $20 \%$, the differences between these two groups were not statistically significant $(p=0.74)$. No significant associations were found between TEM7 and nestin expression in tumor cells and between nestin-MVD and both OS and DFS. Figures 5 and 6 present Kaplan-Meier curves for patients overall survival and DSF in groups with the high, medium and low nestin expression in tumor cells.

\section{DISCUSSION}

Malignant tumor aggressiveness can be determined with the use of various molecular markers. In particular, new angiogenesis markers are of great academic and clinical relevance because a marker-based approach can provide more precise categorization of various cancers. Moreover, new markers themselves can be used as specific targets for developing novel and more effective tumor-targeted therapies. One of the emerging possibilities is a specific blocking of tumor induced microvasculature. Since the role 
Table 2. Nestin expression in tumor cells, MVD-nestin and TEM7 expression correlation with disease free survival (DSF) with overall survival (OS) rates in the studied group of women with HGSOC

\begin{tabular}{|c|c|c|c|c|c|c|c|}
\hline & $\begin{array}{l}\mathrm{N} \text { (no. of } \\
\text { patients) }\end{array}$ & $\begin{array}{c}\text { OS } \\
\text { 3-year }\end{array}$ & $\begin{array}{c}\text { OS } \\
\text { 5-year }\end{array}$ & p & $\begin{array}{c}\text { DSF } \\
\text { 3-year }\end{array}$ & $\begin{array}{c}\text { DSF } \\
\text { 5-year }\end{array}$ & p \\
\hline \multicolumn{8}{|c|}{ Nestin expression in tumor cells } \\
\hline Low & 33 & $42 \%$ & $25 \%$ & \multirow{3}{*}{$p=0.02$} & $23 \%$ & $23 \%$ & \multirow{3}{*}{$p=0.01$} \\
\hline Medium & 23 & $45 \%$ & $24 \%$ & & $16 \%$ & $10 \%$ & \\
\hline High & 14 & $14 \%$ & $0 \%$ & & $9 \%$ & $0 \%$ & \\
\hline \multicolumn{8}{|c|}{ TEM-7 expression in tumor cell } \\
\hline Low & 15 & $28 \%$ & $21 \%$ & \multirow{3}{*}{$p=0.44$} & $8 \%$ & $8 \%$ & \multirow{3}{*}{$p=0.74$} \\
\hline Medium & 26 & $48 \%$ & $19 \%$ & & $21 \%$ & $16 \%$ & \\
\hline High & 29 & $32 \%$ & $25 \%$ & & $20 \%$ & $20 \%$ & \\
\hline \multicolumn{8}{|c|}{ Nestin-MVD SD = 17.3/HPF (min-max 7-30); MD = 16/HPF (range 12-23) } \\
\hline Low $0-15 / \mathrm{HPF}$ & 26 & $44 \%$ & $23 \%$ & \multirow{3}{*}{$p=0.96$} & $17 \%$ & $13 \%$ & \multirow{3}{*}{$p=0.39$} \\
\hline Medium (16-23/HPF) & 27 & $32 \%$ & $28 \%$ & & $21 \%$ & $21 \%$ & \\
\hline High (24-30/HPF) & 17 & $33 \%$ & $11 \%$ & & $11 \%$ & $11 \%$ & \\
\hline
\end{tabular}

OS

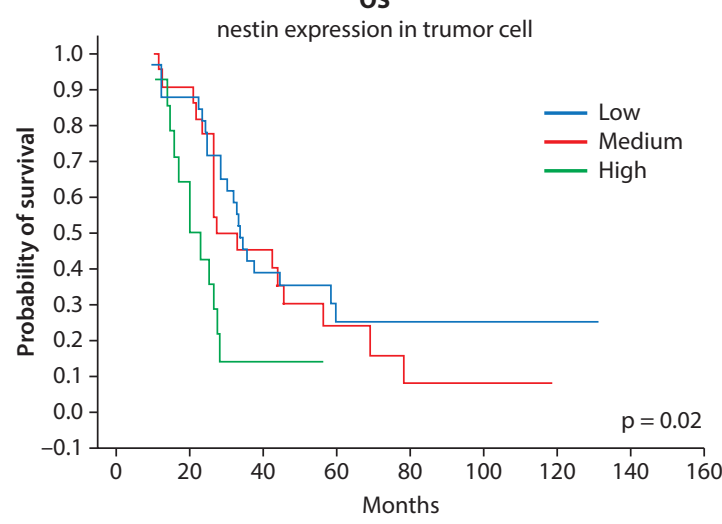

Figure 5. Kaplan-Meier curves for patients overall survival (OS) in the high, medium and low nestin expression groups

of the above mentioned tumors angiogenic markers has not been precisely established to date, we have investigated the expression of TEM7, nestin and nestin-related microvessel density (MVD) in high-grade serous ovarian cancer samples. We have also studied their potential correlations with overall survival and disease-free survival of affected women. The main finding of our study is that nestin expression, but not TEM7 or nestin-MVD angiogenic activity measurements may assist in the prediction of OS and DFS in women with high grade serous ovarian cancer.

In our recent study we have demonstrated that nestin-positive MVD could be used to identify malignant ovarian masses with high angiogenic activity, where tumor vasculature was formed [12]. Qin et al. [20] studied the expression profile of nestin in serous ovarian cancers and found its expression in $32.5 \%$ of cases. The overexpression of

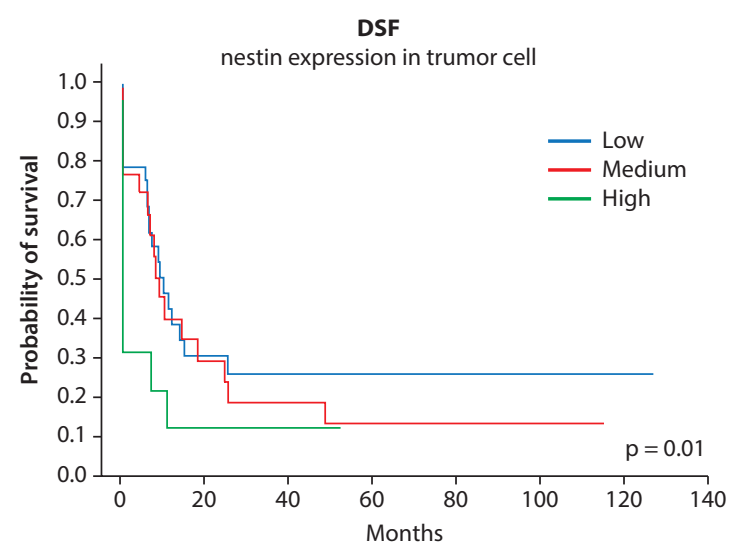

Figure 6. Kaplan-Meier curves for disease free survival (DSF) of women with the high, medium and low nestin expression groups

nestin was associated with the resistance to cisplatin-based chemotherapy and these women had shorter overall survival suggesting that nestin may be a novel and independent prognostic factor. Moreover, when nestin was found in tumor tissue its presence was positively correlated with increased expression of angiogenic markers such as EGFR (Epithelial Growth Factor Receptor), VEGF (Vascular Endothelial Growth Factor) and higher MVD in tumors [20]. He et al. [9] have studied nestin expression evaluated with the use of immunohistochemistry (IHC) in 315 cases of various clinical and histological stages of epithelial ovarian neoplasms as well as in 52 control cases of normal ovarian epithelia. Sal et al. [21] have found increased concentrations of soluble nestin in the serum of ovarian cancer patients. Immunohistochemical results of their study revealed nestin expression was not present in benign masses and gradually increased from early 
borderline to advanced malignant ovarian tumors. These authors have concluded that nestin overexpression was associated with more advanced stage and higher histological grade of serous adenocarcinoma cases [21]. Zhang et al. [22] have suggested that nestin may be significantly associated with the resistance to chemotherapy as in their study the expression of nestin in chemoresistant cells was much higher when compared to parental hepatocellular cancer cells. The origin of nestin-positive vascular endothelial cells in tumor tissue is still controversial. Elevation of nestin expression likely corresponds to reorganization of intermediate filament network in the cytoskeleton of endothelial cells in the course of their maturation or adaptation to changes in growing tissues. The recent study performed by Osmann et al. [10] revealed expression of nestin in almost $50 \%$ of all EOC (Epithelial Ovarian Cancer) cases and it was found most often $(60 \%)$ in serous type of tumors. Taken together these data suggested that nestin could be considered as a prospective therapeutic target for ovarian cancer treatment.

Wang et al. [23] have found that among all the cell surface markers examined in their study only TEM7 expression was up-regulated upon endothelial cells capillary morphogenesis. In addition, inhibition of capillary morphogenesis by serum stimulation completely blocked TEM7 expression. In contrast, stimulation of endothelial cell capillary morphogenesis with PMA enhanced TEM7 expression. Furthermore, incubation of endothelial cells with a recombinant extracellular domain of TEM7 blocked capillary morphogenesis in three-dimensional cultures. These results suggest that TEM7 is a novel protein whose cell surface expression is essential during endothelial cell capillary morphogenesis.

Bagley et al. [14] have investigated TEM7 as a possible target for therapeutic antiangiogenic intervention in cancer. TEM7 expression was assessed by in situ hybridization or by immunohistochemistry (IHC) in 130 formalin-fixed paraffin-embedded (FFPE) and 410 frozen human clinical specimens of cancer and in 301 normal tissue samples. In frozen tumor tissues, TEM7 mRNA and protein was detected in all but one of the cancer types tested and this mRNA was only rarely expressed in the control normal frozen tissues. In FFPE tumor tissues, TEM7 protein was detected by IHC in colon, breast, lung, bladder, ovarian and endometrial cancers and in sarcomas. In contrast, TEM7 protein was not detected in head and neck, prostate or liver cancers. Mehran et al. [24] hypothesized that TEMs may be detectable on circulating endothelial cells (CECs) and that these circulating TEM(+) endothelial cells (CTECs) may be a more specific marker for cancer and tumor response than standard CECs. Following treatment with various vascular-targeting agents, these authors have observed a decrease in CTEC that correlated with the reductions in tumor growth. Novel cancer-associated markers could potentially be used as a blood-based surrogates for assessing the presence of tumor vasculature and antiangiogenic drug activity. Taken together these data indicate that molecular factors that control malignant tumors angiogenesis formation could have a significant implication in future management of patients with advanced clinical stages of HGSOC.

\section{CONCLUSIONS}

Nestin as a marker of cancer stem cells may assist in the prediction of OS and DFS in women with high grade serous ovarian cancer. Nestin may also be considered a novel therapeutic target for antiangiogenic agents.

\section{Authors' contributions}

AC, SC,NS,T , GG and TK had full access to all the data in the study and take responsibility for the integrity of the data, the accuracy of the data analysis, and the decision to submit the manuscript for publication. AC,NS,T, TK and GG conceived, designed and obtained partial funding for the study. SC, NS and AC collected the samples and patient's data. SC performed immunohistochemical studies. SA, NS, T七,TK and GG analyzed the data performer survival analysis and drafted the manuscript. AC supervised the study and revised the manuscript. All authors interpreted the data, critically revised the draft for important intellectual content, and expressed final approval of the manuscript to be published.

\section{Acknowledgments}

This work was supported by grant \#DS119 to AC from the Medical University of Lublin. The Authors wish to thank prof. Jadwiga Kupryjanczyk, the former Head of the Department of Molecular Pathology of the Maria Sklodowska-Curie Memorial Cancer Center and Institute of Oncology, Warsaw, Poland for her kind tissue and data donation and the expertise in tissue samples selection.

\section{REFERENCES}

1. Siegel RL, Miller KD, Jemal A. Cancer Statistics, 2017. CA Cancer J Clin. 2017; 67(1): 7-30, doi: 10.3322/caac.21387, indexed in Pubmed: 28055103.

2. Mittempergher L. Genomic Characterization of High-Grade Serous Ovarian Cancer: Dissecting Its Molecular Heterogeneity as a Road Towards Effective Therapeutic Strategies. Curr Oncol Rep. 2016; 18(7): 44, doi: 10.1007/s11912-016-0526-9, indexed in Pubmed: 27241520.

3. McCluggage WG. Morphological subtypes of ovarian carcinoma: a review with emphasis on new developments and pathogenesis. Pathology. 2011; 43(5): 420-432, doi: 10.1097/PAT.0b013e328348a6e7, indexed in Pubmed: 21716157.

4. Schmid BC, Oehler MK. New perspectives in ovarian cancer treatment Maturitas. 2014; 77(2): 128-136, doi: 10.1016/j.maturitas.2013.11.009, indexed in Pubmed: 24380827.

5. Karakaya BK, Başer E, Bildacı B, et al. Alternative tumor markers in the diagnosis of ovarian cancer. Ginekol Pol. 2016; 87(8): 565-769, doi: 10.5603/GP.2016.0045, indexed in Pubmed: 27629130.

6. Jain RK. Normalization of tumor vasculature: an emerging concept in antiangiogenic therapy. Science. 2005; 307(5706): 58-62, doi: 10.1126/science.1104819, indexed in Pubmed: 15637262. 
7. Markowska A, Sajdak S, Markowska J, et al. Angiogenesis and cancer stem cells: New perspectives on therapy of ovarian cancer. Eur J Med Chem. 2017; 142: 87-94, doi: 10.1016/j.ejmech.2017.06.030, indexed in Pubmed: 28651817.

8. Croix B, Rago C, Velculescu V, et al. Genes Expressed in Human Tumor Endothelium. Science. 2000; 289(5482): 1197-1202, doi: 10.1126/science.289.5482.1197.

9. He QZ, Luo XZ, Zhou Q et al. Expression of nestin in ovarian serous cancer and its clinicopathologic significance. Eur Rev Med Pharmacol Sci. 2013; 17(21): 2896-2901, indexed in Pubmed: 24254558.

10. Neradil J, Veselska R. Nestin as a marker of cancer stem cells. Cancer Sci. 2015; 106(7): 803-811, doi: 10.1111/cas.12691, indexed in Pubmed: 25940879.

11. Osman WM, Shash LS, Ahmed NS. Emerging Role of Nestin as an Angiogenesis and Cancer Stem Cell Marker in Epithelial Ovarian Cancer: Immunohistochemical Study. Appl Immunohistochem Mol Morphol. 2017; 25(8): 571-580, doi: 10.1097/PAl.0000000000000338, indexed in Pubmed: 26945442.

12. Czekierdowska S, Stachowicz N, Chróściel M, et al. Proliferation and maturation of intratumoral blood vessels in women with malignant ovarian tumors assessed with cancer stem cells marker nestin and platelet derived growth factor PDGF-B. Ginekol Pol. 2017; 88(3): 120-128, doi: 10.5603/GP.a2017.0023, indexed in Pubmed: 28397199.

13. Svachova H, Kryukov F, Kryukova E, et al. Nestin expression throughout multistep pathogenesis of multiple myeloma. Br J Haematol. 2014; 164(5): 701-709, doi: 10.1111/bjh.12689, indexed in Pubmed: 24329895.

14. Bagley RG, Rouleau C, WeberW, et al. Tumor endothelial marker 7 (TEM7): a novel target for antiangiogenic therapy. Microvasc Res. 2011;82(3): 253-262, doi: 10.1016/j.mvr.2011.09.004, indexed in Pubmed: 21958527.

15. Pietrzyk Ł. Biomarkers Discovery for Colorectal Cancer: A Review on Tumor Endothelial Markers as Perspective Candidates. Dis Markers. 2016; 2016: 4912405, doi: 10.1155/2016/4912405, indexed in Pubmed: 27965519.
16. Zhang ZZ, Hua R, Zhang JF, et al.TEM7 (PLXDC1), a key prognostic predictor for resectable gastric cancer, promotes cancer cell migration and invasion. Am J Cancer Res. 2015; 5(2): 772-781, indexed in Pubmed: 25973314

17. Nanda A, Buckhaults $P$, Seaman $S$, et al. Identification of a binding partner for the endothelial cell surface proteins TEM7 and TEM7R. Cancer Res. 2004; 64(23): 8507-8511, doi: 10.1158/0008-5472.CAN-04-2716, indexed in Pubmed: 15574754

18. Fuchs $B$, Mahlum $E_{1}$ Halder $C$, et al. High expression of tumor endothelial marker 7 is associated with metastasis and poor survival of patients with osteogenic sarcoma. Gene. 2007; 399(2): 137-143, doi: 10.1016/j. gene.2007.05.003, indexed in Pubmed: 17560052.

19. Kupryjanczyk J, Kraszewska E, Ziolkowska-Seta I, et al. TP53 status and taxane-platinum versus platinum-based therapy in ovarian cancer patients: A non-randomized retrospective study. BMC Cancer. 2008; 8 , doi: 10.1186/1471-2407-8-27.

20. Qin $Q$, Sun $Y$, Fei $M$, et al. Expression of putative stem marker nestin and CD133 in advanced serous ovarian cancer. Neoplasma. 2012; 59(3): 310-315, doi: 10.4149/neo 2012 040, indexed in Pubmed: 22296500.

21. Sal V, Kahramanoglu I, Bese T, et al. Is serum level of nestin useful in detecting epithelial ovarian cancer? J Obstet Gynaecol Res. 2017; 43(2): 371-377, doi: 10.1111/jog.13220, indexed in Pubmed: 27995729.

22. Zhang Y, Zeng S, Ma J, et al. Nestin overexpression in hepatocellular carcinoma associates with epithelial-mesenchymal transition and chemoresistance. J Exp Clin Cancer Res. 2016; 35(1): 111, doi: 10.1186/s13046016-0387-y, indexed in Pubmed: 27412382.

23. Wang XQ, Sheibani N, Watson JC. Modulation of tumor endothelial cell marker 7 expression during endothelial cell capillary morphogenesis. Microvasc Res. 2005; 70(3): 189-197, doi: 10.1016/j.mvr.2005.08.004, indexed in Pubmed: 16202431.

24. Mehran R, Nilsson M, Khajavi M, et al. Tumor endothelial markers define novel subsets of cancer-specific circulating endothelial cells associated with antitumor efficacy. Cancer Res. 2014; 74(10): 2731-2741, doi: 10.1158/0008-5472.CAN-13-2044, indexed in Pubmed: 24626092. 